Conclusion: Substantial heterogeneity exists among registries regarding $A E$ assessment within the JAK-pot collaboration. These differences must be taken into account when analysing the safety of JAKi across different countries in collaborative studies. For comparative analyses, stratified analyses by country are required to account for differential $A E$ assessment and varying degrees of potential under-reporting.

Disclosure of Interests: Kim Lauper: None declared, Denis Mongin: None declared, Sytske Anne Bergstra: None declared, Denis Choquette: None declared, Catalin Codreanu: None declared, Diederik De Cock: None declared, Lene Dreyer: None declared, Ori Elkayam: None declared, Kimme Hyrich: None declared, Florenzo lannone: None declared, Nevsun Inanc: None declared, Eirik kristianslund: None declared, Tore K. Kvien: None declared, Burkhard Leeb: None declared, Galina Lukina: None declared, Dan Nordström: None declared, Karel Pavelka: None declared, Manuel Pombo-Suarez: None declared, Ziga Rotar: None declared, Maria Jose Santos: None declared, Anja Strangfeld: None declared, Delphine Courvoisier: None declared, Axel Finckh Speakers bureau: Eli-Lilly, Pfizer, Consultant of: Eli-Lilly, Pfizer, Grant/research support from: BMS, Pfizer.

DOI: 10.1136/annrheumdis-2021-eular.2216

\section{POS0094 EFFECTS OF RANKL INHIBITION ON PROMOTING HEALING OF BONE EROSION IN RHEUMATOID ARTHRITIS USING HR-PQCT: A 2-YEAR, RANDOMIZED, DOUBLE-BLIND, PLACEBO-CONTROLLED TRIAL}


J. F. Griffith ${ }^{3}$, V. W. Lee ${ }^{4}$, L. Shi ${ }^{3}$, J. Huang ${ }^{3}$, Y. K. Kwok ${ }^{5}$, I. C. Yim ${ }^{6}$, T. K. LI ${ }^{1}$, V. Lo ${ }^{1}$, J. M. Lee ${ }^{7}$, J. J. W. Lee ${ }^{8}$, L. Qin ${ }^{2}$, L. S. Tam ${ }^{1} .{ }^{1}$ The Chinese University of Hong Kong, Medicine and Therapeutics, Hong Kong, Hong Kong (SAR); ${ }^{2}$ The Chinese University of Hong Kong, Orthopedics \& Traumatology, Hong Kong, Hong Kong (SAR); ${ }^{3}$ The Chinese University of Hong Kong, Imaging \& Interventional Radiology, Hong Kong, Hong Kong (SAR); ${ }^{4}$ The Chinese University of Hong Kong, School of Pharmacy, Hong Kong, Hong Kong (SAR); ${ }^{5}$ Queen Elizabeth Hospital, Medicine, Hong Kong, Hong Kong (SAR); ${ }^{6}$ Tseung Kwan O Hospital, Medicine, Hong Kong, Hong Kong (SAR); ${ }^{7}$ Tai Po Hospital, Medicine and Geriatrics, Hong Kong, Hong Kong (SAR); ${ }^{8}$ The Chinese University of Hong Kong, The Jockey Club School of Public Health \& Primary Care, Hong Kong, Hong Kong (SAR)

Background: Partial repair of bone erosions in rheumatoid arthritis (RA) is known from high-resolution peripheral quantitative computer tomography (HR-pQCT) studies in patients with moderate to high disease activity using biologics [1]. Whether RANKL inhibition by denosumab is efficacious in healing existing erosions in RA patients with low disease activity or in remission on conventional synthetic DMARDs is uncertain.

Objectives: To evaluate the effects of denosumab on erosion healing at 2-4 metacarpophalangeal head as determined by HR-pQCT in patients with RA with stable disease.

Methods: This was a randomized, placebo-controlled, double-blind study. RA patients with disease activity score 28 joints (DAS28) $\leq 5.1$ were randomized $(1: 1)$ to subcutaneous denosumab $60 \mathrm{mg}$ or placebo once every six months for 24 months. The primary outcome was erosion healing at MCP 2-4 on HR-pQCT at 12 months. The effects of denosumab on erosion and joint space parameters on HR-pQCT and radiographs, disease activity and health assessment questionnaire-disability index (HAQ-DI) were also examined.

Results: At 24 months, HR-pQCT images were analyzed in 98 patients. Baseline demographic, clinical characteristics and imaging parameters were comparable between the two treatment groups (table 1). Seventeen patients in each group (placebo group: 17/52, 32.6\%; denosumab group: 17/50, $34.0 \%$ ) achieved sustained low disease activity (DAS28 $\leq 3.2$ ) throughout the 24 months. At 12 months, changes in erosion parameters on HR-pQCT were similar between the two groups. At 24 months, new erosions (19\% vs $9 \%, p=0.009)$ and erosion progression ( $34 \%$ vs $16 \%, p<0.001)$ were more common in the placebo group than the denosumab group. Erosion healing was seen in a significantly higher proportion of patients in the denosumab group $(20 \%$ vs $6 \%, p=0.045)$ at 24 months. The details of the changes in HR-pQCT erosion parameters are shown in figure 1. No significant differences in the changes in joint space parameters on HR-pQCT, van der Heijde-Sharp erosion score, DAS28 and HAQ-DI were observed between the two groups at 12 and 24 months.

Conclusion: Although no differences in erosion parameters were observed at 12 months, denosumab was more efficacious than placebo in erosion repair on HR-pQCT after 24 months.

REFERENCES:

[1] Finzel S, Rech J, Schmidt S, et al. Interleukin-6 receptor blockade induces limited repair of bone erosions in rheumatoid arthritis: a micro CT study. Ann Rheum Dis 2013;72:396-400.
Table 1. Baseline clinical, demographic, disease activity parameters and medications

\begin{tabular}{|c|c|c|c|}
\hline & Placebo $(n=55)$ & Denosumab $(n=55)$ & Total $(n=110)$ \\
\hline $\begin{array}{l}\text { Age } \\
\text { Gender (Female) }\end{array}$ & $\begin{array}{c}56.5 \pm 7.1 \\
47(86)\end{array}$ & $\begin{array}{c}57.2 \pm 8.5 \\
41(75)\end{array}$ & $\begin{array}{c}56.8 \pm 7.8 \\
88(80)\end{array}$ \\
\hline Disease duration (years) & $8.5 \pm 6.8$ & $7.3 \pm 6.9$ & $7.9 \pm 6.8$ \\
\hline Rheumatoid factor positive & $40(72)$ & $38(69)$ & $78(71)$ \\
\hline ACPA positive & $43(78)$ & $44(80)$ & $87(79)$ \\
\hline DAS28-CRP & $2.43 \pm 0.83$ & $2.6 \pm 0.92$ & $2.51 \pm 0.88$ \\
\hline DAS28-CRP>3.2 & $8(15)$ & $13(24)$ & $21(19)$ \\
\hline HAQ-DI (0-3) & $0.31 \pm 0.38$ & $0.46 \pm 0.47$ & $0.39 \pm 0.43$ \\
\hline csDMARDs & $49(89)$ & $52(95)$ & $101(92)$ \\
\hline Combination csDMARDs & $26(47)$ & $33(60)$ & $59(54)$ \\
\hline Glucocorticoids & $5(10)$ & $5(9)$ & $10(9)$ \\
\hline vdH- Sharp erosion score & $10.4 \pm 18.4$ & $8.9 \pm 13.8$ & $9.6 \pm 16.2$ \\
\hline vdH- Sharp JSN score & $12.4 \pm 17.7$ & $11.5 \pm 17.2$ & $11.9 \pm 17.4$ \\
\hline Lumbar spine $\mathrm{aBMD}, \mathrm{g} / \mathrm{cm}^{2}$ & $0.914 \pm 0.147$ & $0.930 \pm 0.143$ & $0.922 \pm 0.145$ \\
\hline Total hip aBMD, $\mathrm{g} / \mathrm{cm}^{2}$ & $0.837 \pm 0.102$ & $0.847 \pm 0.146$ & $0.841 \pm 0.125$ \\
\hline Femoral neck aBMD, $\mathrm{g} / \mathrm{cm}^{2}$ & $0.681 \pm 0.099$ & $0.695 \pm 0.128$ & $0.687 \pm 0.114$ \\
\hline
\end{tabular}

Data are reported as mean \pm SD or number (\%). ACPA: Anti-cyclic citrullinated peptide antibody; DAS28: disease activity score 28; csDMARDs: conventional synthetic disease modifying anti-rheumatic drug. HAQ-DI: health assessment questionnaire disability index; vdH- Sharp score: Van der Heijde- Sharp score; aBMD: areal bone mineral density
A.

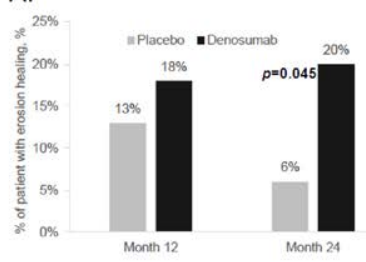

C.

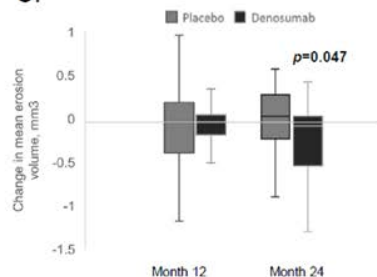

E.

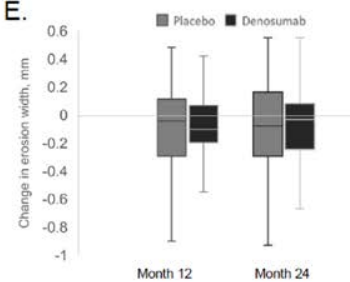

G.

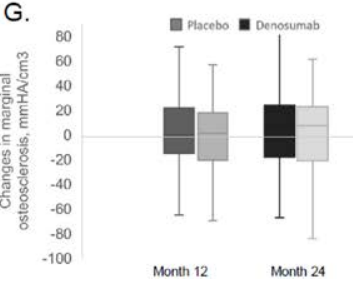

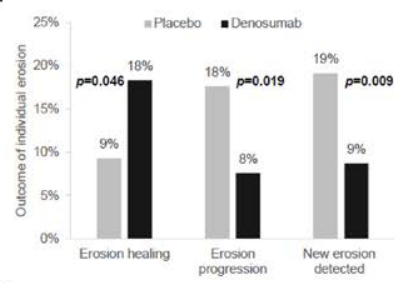

D.



F.

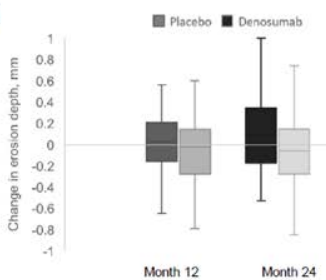

Figure 1. Changes in erosion parameters by HR-pQCT. (A) Percentage of patients with overal erosion healing; (B) Outcome of individual erosion with healing, progression and new erosion detected across study period; change in (C) mean erosion volume; (D) total erosion volume; (E) erosion width; $(\mathrm{F})$ erosion depth and $(\mathrm{G})$ marginal osteosclerosis per patient.

Disclosure of Interests: Ho SO: None declared, Isaac T. Cheng: None declared, Sze-Lok Lau: None declared, Evelyn Chow: None declared, Tommy Lam: None declared, Vivian W Hung: None declared, Edmund Li: None declared, James F Griffith: None declared, Vivian WY Lee: None declared, Lin Shi: None declared, Junbin Huang: None declared, Yan Kitty Kwok: None declared, Isaac C Yim: None declared, Tena K. Li: None declared, Vincent Lo: None declared, Jolly M Lee: None declared, Jack Jock Wai Lee: None declared, Ling Qin: None declared, LaiShan Tam Grant/research support from: Grants from Novartis and Pfizer DOI: 10.1136/annrheumdis-2021-eular.2752 